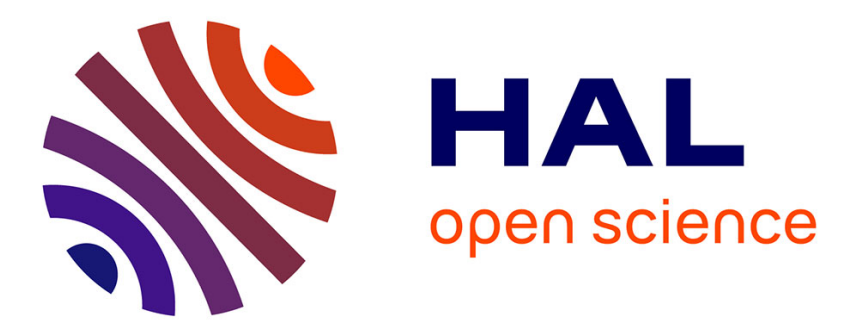

\title{
La résistance du piment a Phytophthora capsici. XIII: Mise en évidence d'une induction de résistance par des extraits de jeunes piments contaminés ou élicités
} Paul-Michel Molot, Pierre Mas

\section{- To cite this version:}

Paul-Michel Molot, Pierre Mas. La résistance du piment a Phytophthora capsici. XIII: Mise en évidence d'une induction de résistance par des extraits de jeunes piments contaminés ou élicités. Agronomie, 1986, 6 (2), pp.213-217. 10.1051/agro:19860211 . hal-02728702

\section{HAL Id: hal-02728702 \\ https://hal.inrae.fr/hal-02728702}

Submitted on 2 Jun 2020

HAL is a multi-disciplinary open access archive for the deposit and dissemination of scientific research documents, whether they are published or not. The documents may come from teaching and research institutions in France or abroad, or from public or private research centers.
L'archive ouverte pluridisciplinaire HAL, est destinée au dépôt et à la diffusion de documents scientifiques de niveau recherche, publiés ou non, émanant des établissements d'enseignement et de recherche français ou étrangers, des laboratoires publics ou privés. 


\title{
La résistance du piment à Phytophthora capsici. XIII. - Mise en évidence d'une induction de résistance par des extraits de jeunes piments contaminés ou élicités
}

Paul-Michel MOLOT \& Pierre MAS

I.N.R.A., Station de Pathologie végétale, Centre de Recherches agronomiques d'Avignon, F 84140 Montfavet

RÉSUMÉ

\begin{abstract}
Des extraits, obtenus par infiltration d'eau dans les tissus, sont préparés à partir de plantules de piment soit saines, soit contaminées par pulvérisation de spores de Phytophthora capsici, soit élicitées par trempage des racines dans la fraction G5 15. Déposés sur cotylédons en survie de piment «Yolo Wonder », ils peuvent induire une résistance vis-à-vis de $P$. capsici.

Les extraits de piment résistant à $P$. capsici, «Phyo 636 », sont toujours plus efficaces que les extraits de piment sensible, « Yolo Wonder ».

L'effet de protection, déjà net avec des extraits de matériel sain, est accentué avec des extraits de matériel contaminé ou élicité.

N'ayant pas d'action inhibitrice sur la germination in vitro des zoospores de $P$. capsici, mais déclenchant au contact du végétal une induction de résistance, les extraits obtenus peuvent être considérés comme des éliciteurs endogènes.
\end{abstract}

L'intérêt des éliciteurs endogènes et exogènes est discuté.

Mots clés additionnels : Eliciteur exogène, éliciteur endogène, germination de spores in vitro, liquide intercellulaire, résistance quantitative.

Resistance of Capsicum annuum to Phytophthora capsici. XIII. - Induced resistance by intercellular fluid from young plants of pepper infected or elicited.

Extracts, obtained by infiltration of water into tissues, were prepared from young peppers (healthy or infected by Phytophthora capsici or elicited by the elicitor fraction G5 15). These extracts, placed on detached pepper cotyledons, induced resistance to $P$. capsici. Extracts from resistant pepper, "Phyo 636", were always more active than those from susceptible pepper, "Yolo Wonder". The protection effect, already high with extracts from healthy plants, increased with those from infected or elicited plants. The extracts had no inhibitory effect on zoospore germination; they behaved as endogenous elicitors. The concept of endogenous and exogenous elicitors is discussed.

Additional key words : Exogenous elicitor, endogenous elicitor, in vitro spore germination, intercellular fluid, quantitative resistance.

\section{INTRODUCTION}

Les phytoalexines sont des composés antifongiques produits par les plantes en réponse à une attaque par un microorganisme ou à l'action d'un traitement nocif (agent chimique, traumatisme, choc thermique, radiations ultra-violettes, etc.). Les mécanismes qui président à la formation et à l'accumulation de phytoalexines dans les tissus végétaux ne sont pas encore conve- nablement élucidés. On a suggéré que des éliciteurs, c'est-à-dire des substances excrétées par l'agent pathogène, étaient responsables de l'induction de synthèse des phytoalexines lors d'interactions spécifiques dans des sites complémentaires de la plante hôte.

Avec le couple haricot-Colletotrichum lindemuthianum, la destruction des tissus infectés s'accompagne souvent de l'accumulation de diverses phytoalexines qui se forment dès la mort des cellules et se concentrent uniquement à ce niveau (BAILEY, 1974). 
Les éliciteurs abiotiques (bichlorure de mercure, chloroforme), les toxines, les dégâts physiques (congélation, dégel), les infections microbiennes et probablement les éliciteurs biotiques engendrent des altérations qui aboutissent à la mort des cellules. Un métabolite, appelé éliciteur constitutif (ou endogène), est alors libéré par les cellules mortes; il initie dans les tissus adjacents vivants la synthèse de novo de phytoalexines. Celles-ci diffusent des tissus vivants vers les cellules mortes où elles s'accumulent à des concentrations très élevées (BAILEY, 1980, 1982). Nous-mêmes, avec le couple piment Phytophthora capsici Leon., avons pu mettre en évidence de plus fortes concentrations en capsidiol dans les zones nécrotiques que dans les zones non nécrotiques (MOLOT et al., 1982), toutes deux étant envahies par le parasite.

Différents travaux ont montré que l'application à des hypocotyles (HARGREAVES \& BAILEY, 1978) ou à des suspensions cellulaires de haricot (HARGREAVES \& SELBY, 1978) d'extraits de tissus de cette même plante, ayant subi ou non un traumatisme, pouvait déclencher la synthèse de phytoalexines. De plus, par autoclavage à chaud ou par hydrolyse acide de parois cellulaires de soja, on a pu isoler un polysaccharide biologiquement actif sur cotylédons de cette même plante et renfermant rhamnose, xylose et acide galacturonique (HAHN et al., 1981). Des substances identiques ont été décelées dans les parois de cellules en suspension liquide de tabac, érable et blé. Plus récemment, un éliciteur provenant de tiges de soja congelées, vraisemblablement une enzyme thermolabile de nature glycoprotéinique, s'est révélé inducteur de phytoalexines (LYON \& ALBERSHEIM, 1982). Ces études suggèrent la présence, chez ces plantes, d'éliciteurs endogènes.

Dans le travail présenté ici, nous nous proposons de mettre en évidence le pouvoir inducteur de résistance chez Capsicum annuum L. d'extraits de plantules de piment soit saines, soit contaminées, soit soumises à l'action d'un éliciteur exogène d'origine fongique.

\section{MATÉRIEL ET MÉTHODES}

\section{A. Préparation du matériel végétal}

Deux variétés de piment, l'une sensible, «Yolo Wonder », l'autre résistante, «Phyo 636 », à $P$. capsici, sont semées en terrines et élevées en serre jusqu'au stade 2 cotylédons - 1 feuille. Ce matériel est alors soit contaminé soit élicité.

\section{Contamination par $\mathrm{P}$. capsici}

Une suspension de zoospores de la souche $15 \mathrm{de}$ $P$. capsici ajustée à 40000 zoospores/ml est pulvérisée à la surface du feuillage. Le matériel est ensuite placé en chambre humide, sous éclairage artificiel $(16 \mathrm{~h}$ d'éclairement par jour) pendant $4 \mathrm{j}$ à $18-22{ }^{\circ} \mathrm{C}$. $\mathrm{Au}$ bout de ce laps de temps, les plantes sont prélevées. Les symptômes n'apparaissent que vers le $5^{\text {e }}$ ou le $6^{\mathrm{e}}$ jour : au niveau du feuillage, on observe chez la variété sensible de larges plages nécrotiques, chez la variété résistante de fines ponctuations jaunâtres.
Les plantules témoins sont pulvérisées avec de l'eau et soumises aux mêmes conditions expérimentales.

\section{Elicitation}

Les plantules sont arrachées et leurs racines, après lavage, sont trempées pendant $48 \mathrm{~h}$ selon une technique déjà décrite (MOLOT \& MAS, 1985), dans une fraction hydrosoluble G5 15 issue du filtrat de culture de la souche 15 de $P$. caspici (MOLOT et al., 1980). La concentration en G5 15 est de $0,25 \mathrm{mg} / \mathrm{ml}$ d'eau. Les plantules témoins sont trempées dans l'eau pendant le même temps.

\section{B. Prélèvement du matériel végétal et obtention des extraits}

Après élimination des racines, la totalité du système aérien des plantes témoins, infectées ou élicitées (tiges et feuilles) est coupée en menus morceaux et immergée dans l'eau à raison de $8 \mathrm{~g}$ de matériel frais pour $100 \mathrm{ml}$ d'eau distillée. L'ensemble est soumis à un vide de 0,90 bar ; le retour à la pression atmosphérique commence au bout de $10 \mathrm{mn}$ et s'effectue très lentement pendant environ $1 \mathrm{~h}$; les espaces interstitiels, normalement occupés par de l'air, sont désormais remplis d'eau.

Afin de récupérer ce liquide d'infiltration, le matériel végétal est soigneusement essuyé, puis centrifugé pendant $10 \mathrm{mn}$ à 6000 RPM dans des godets dont le fond est garni d'une grille (DE WITT \& SPIKMAN, 1982). Le rendement en liquide d'infiltration est d'environ 25 p. 100 ( $2 \mathrm{ml}$ pour $8 \mathrm{~g}$ de matière fraîche). Le liquide d'infiltration est alors conservé au congélateur à $-20^{\circ} \mathrm{C}$.

\section{Contrôles biologiques des extraits obtenus}

\section{Pouvoir germinatif in vitro des zoospores de P. capsici dans ces extraits}

Des zoospores de la souche 15 sont ajoutées dans chaque extrait à raison de $0,1 \mathrm{ml}$ de suspension de zoospores pour $0,25 \mathrm{ml}$ d'extrait (62 500 zoospores $/ \mathrm{ml}$ de préparation finale).

Les lectures s'effectuent $3 \mathrm{~h}$ après la mise en germination à $22^{\circ} \mathrm{C}$. Elles consistent à apprécier au microscope le pourcentage de zoospores germées et la longueur des tubes germinatifs (LTG). Celle-ci est déterminée en ne tenant compte que des seules zoospores germées.

\section{Pouvoir éliciteur des extraits sur cotylédons de piment « Yolo Wonder »}

Des cotylédons frais sont mis en survie sur l'eau. Sur la face inférieure (non blessée), tournée vers le haut, on dépose $25 \mu \mathrm{l}$ d'extraits, puis $24 \mathrm{~h}$ après et sur le même site, $10 \mu$ l d'une suspension de zoospores de la souche 15 (62 500 zoospores/ml). Après $3 \mathrm{j}$ d'incubation à $22{ }^{\circ} \mathrm{C}$ sous $16 \mathrm{~h}$ de lumière, les symptômes apparaissent ; ils sont notés selon une méthode déjà décrite qui permet d'apprécier la sensibilité en pourcentages (MOLOT et al., 1980). Chaque traitement comporte 20 cotylédons. 


\section{RÉSULTATS}

\section{A. Germination in vitro des zoospores de $\boldsymbol{P}$. capsici dans les différents extraits (tabl. 1)}

Dans le témoin eau, la germination des zoospores est faible $(24$ p. 100$)$; en revanche dans la fraction élicitrice G5 15 et surtout dans les différents extraits, le pourcentage de germination est nettement plus élevé ; ce résultat peut s'expliquer par une plus grande richesse du milieu en éléments nutritifs ainsi que semble l'indiquer les résultats de germination dans du milieu nutritif synthétique. On remarquera que les extraits de "Yolo Wonder » sont plus stimulants que ceux de «Phyo 636 » mais, pour une variété donnée, il n'y a pas de différence significative entre les extraits. Ces résultats sont confirmés par les mesures des longueurs de tubes germinatifs.

\section{B. Sensibilité à $P$. capsici des cotylédons de piment traités par différents extraits (tabl. 1)}

Dans nos conditions expérimentales, le témoin « éliciteur » G5 15 protège assez faiblement les cotylédons de "Yolo Wonder ». Par contre, les extraits de piment dont les racines ont absorbé cette fraction assurent une protection partielle lorsqu'ils proviennent de "Yolo Wonder " et totale lorsqu'ils proviennent de "Phyo 636 ». Chez "Yolo Wonder », l'effet paraît encore plus accentué quand les extraits sont préparés à partir de plantules contaminées. De plus, on remarquera que les extraits de plantules témoins (témoin contamination, témoin élicitation) sont euxmêmes inducteurs de résistance, mais à un degré moindre. D'une façon générale, les extraits de «Phyo 636 » protègent mieux que ceux de «Yolo Wonder ».

\section{DISCUSSION ET INTERPRÉTATION}

Comme nous l'avons déjà indiqué, des travaux récents ont montré que des extraits de plantes saines, obtenus généralement dans des conditions assez sévères (bichlorure de mercure, congélation et décongélation successive, autoclavage à chaud, hydrolyse acide) sont capables de favoriser l'accumulation de phytoalexines et d'induire une réaction de défense chez le végétal traité.

Dans le travail présenté ici, nous montrons que les extraits de plantules saines de piment, préparés par une méthode relativement douce, possèdent aussi une bonne activité élicitrice. De plus, deux faits sont à remarquer :

- les extraits de piments contaminés ont une activité élicitrice supérieure à celle des extraits de piments sains. Ce résultat confirme des travaux antérieurs où les extraits préparés à partir de tiges de piment adulte contaminées par le test décapitation s'étaient révélés efficaces (MICHEL \& MOLOT, 1980);

- les extraits de piment provenant de plantes ayant absorbé par leurs racines une fraction élicitrice hydrosoluble possèdent une activité élicitrice comparable à celle des extraits de plantes contaminées. A notre connaissance, ce point n'avait pas encore été signalé.

Ces résultats ont été obtenus dans 3 expériences successives réalisées chaque fois dans les mêmes conditions. Celles-ci, décrites au chapitre II, ont été définies dans des tests préliminaires montrant qu'il n'y avait pas de différence entre des extraits issus de plantules d'âge légèrement différent ( 1 ou 3 feuilles bien développées) et que les prélèvements effectués au $4^{\mathrm{e}} \mathrm{j}$ (après élicitation ou contamination) étaient préférables à ceux $d u 2^{e} \mathrm{j}$. La concentration en extraits retenue $(0,25 \mathrm{ml} / \mathrm{g}$ de matière fraîche) correspond à la

Influence du liquide intercellulaire de 2 variétés de piment, «Yolo Wonder »(YW) et « Phyo 636 », saines, élicitées par G5 15 ou contaminées par Phytophthora capsici (P.c.) sur la germination in vitro des zoospores et sur la sensibilité des cotylédons de YW à ce parasite.

Influence of intercellular fluid from 2 pepper cultivars, Yolo Wonder (YW) and Phyo 636, healthy, elicited by G5 15 fraction or inoculated with Phytophthora capsici (P.c.), on in vitro zoospore germination and on susceptibility of $Y W$ cotyledons to the pathogen.

\begin{tabular}{|c|c|c|c|c|}
\hline & & \multicolumn{2}{|c|}{$\begin{array}{l}\text { Germination in vitro } \\
\text { des zoospores de P.c. }\end{array}$} & \multirow{2}{*}{$\begin{array}{l}\text { Sensibilité en p. } 100 \\
\text { à P.c. des cotylédons } \\
\text { de YW }\end{array}$} \\
\hline & & $\begin{array}{l}\text { p. } 100 \\
\text { germination }\end{array}$ & $\begin{array}{l}\mathrm{LTG} * \\
\text { en } \mu \mathrm{m}\end{array}$ & \\
\hline Extrait de «YW» & $\begin{array}{l}\text { - témoin (contamination) } \\
\text { - témoin (élicitation) } \\
\text { - élicité par G5 } 15 \\
\text { - contaminé par P.c. }\end{array}$ & $\begin{array}{l}91 \pm 5,3 \\
90 \\
92 \\
95\end{array}$ & $\begin{array}{l}68 \pm 3,7 \\
63 \\
63 \\
62\end{array}$ & $\begin{array}{l}75 \pm 3,2 \\
79 \\
43 \\
36\end{array}$ \\
\hline Extrait de «Phyo $636 »$ & $\begin{array}{l}\text { - témoin (contamination) } \\
\text { - témoin (élicitation) } \\
\text { - élicité par G5 } 15 \\
\text { - contaminé par } P . c .\end{array}$ & $\begin{array}{l}70 \\
72 \\
73 \\
69\end{array}$ & $\begin{array}{l}49 \\
48 \\
44 \\
46\end{array}$ & $\begin{array}{r}29 \\
26 \\
0 \\
0\end{array}$ \\
\hline $\begin{array}{l}\text { Témoin éliciteur G5 } 15 \\
\text { Témoin milieu nutritif } \\
\text { Témoin eau }\end{array}$ & $\begin{array}{l}(0,25 \mathrm{mg} / \mathrm{ml}) \\
\text { étique }\end{array}$ & $\begin{array}{l}36 \\
95 \\
24\end{array}$ & $\begin{array}{l}29 \\
71 \\
23\end{array}$ & $\begin{array}{r}83 \\
98 \\
100\end{array}$ \\
\hline
\end{tabular}

* LTG $=$ longueur du tube germinatif. 
concentration optimum en deçà et au-delà de laquelle l'effet éliciteur diminue (MOLOT et al., 1983 ; MOLOT, 1984). Enfin, pour des raisons de commodité (dose d'inoculum plus faible, lecture des symptômes plus aisée), les tests de contrôle sont toujours réalisés sur cotylédons de "Yolo Wonder ». L'éliciteur G5, assez peu efficace quant à son effet local, doit être mis au contact des racines pour donner, après prélèvement de la partie aérienne, des extraits actifs sur cotylédons de «Yolo Wonder».

Il semble donc que l'éliciteur « intéressant » ne soit pas la fraction G5, mais un produit élaboré par la plante soit au contact du parasite, soit au contact d'un métabolite produit in vitro par ce même parasite. Ce produit, existant peut-être à l'état de traces dans les extraits de plantes saines, verrait sa synthèse accrue après contamination ou élicitation.

Les extraits obtenus peuvent être considérés comme des éliciteurs endogènes. Pour être actifs, ceux-ci doivent rester au contact des tissus du végétal. In vitro, ils n'empêchent pas la germination des zoospores; celles-ci germent d'ailleurs très bien dans les gouttes infectieuses déposées, lors des tests sur cotylédons, au site même d'élicitation ; la mise en place de mécanismes de défense est alors matérialisée par une pénétration difficile des tubes germinatifs, voire même par l'absence totale de pénétration.

Actuellement, on dispose de peu de données précises sur la composition des éliciteurs endogènes. On pense qu'il s'agit de composés à faible poids moléculaire (HARGREAVES \& BAILEY, 1978) ; un polysaccharide, où l'acide galacturonique paraît être le facteur essentiel de l'activité élicitrice, a été isolé des parois cellulaires du soja (HAHN et al., 1981). Récemment on a apporté quelques éléments en faveur de la nature enzymatique des éliciteurs endogènes (LYON \& ALBERSHEIM, 1982); une $\beta$ 1-3 glucanase isolée du soja est capable, après mise en contact avec le mycélium de Phytophthora megasperma var. sojae, de donner un glucomannane actif (KEEN et al., 1983) ; des extraits de haricot résistant à $C$. lindemuthianum, obtenus par voie enzymatique ou chimique et confrontés à un haricot sensible ou résistant, déclenchent la synthèse d'une $\beta$ 1-3 glucanase qui dégrade directement les parois du champignon (BARTHE, 1984).

Dans l'interaction hôte-parasite, les éliciteurs exogènes microbiens produits in vitro et surtout les éliciteurs endogènes que l'hôte émet sous l'influence du parasite pourraient concourir à la mise en place des mécanismes de défense.

Le principal intérêt des éliciteurs exogènes serait de provoquer le "relargage " des éliciteurs endogènes (OuCHI, 1983). Ceux-ci, en induisant une accumulation localisée de phytoalexines ou en étant susceptibles d'être véhiculés par les tissus (caractère systémique de la résistance), pourraient amplifier les réactions de défense et jouer ainsi un rôle déterminant.

La spécificité d'action des éliciteurs endogènes pourrait être beaucoup plus grande que celle des éliciteurs exogènes. A ce propos, il a été montré que seuls les extraits de haricot résistant à $C$. lindemuthianum sont en mesure d'induire au contact du végétal, l'émission d'une enzyme de défense (BARTHE, 1984).

L'expression de remarquables phénomènes d'immunité dans les conditions de la pratique (KUĆ, 1982) demeure sans explication à l'heure actuelle. Il y a, peut-être, intervention d'éliciteurs endogènes, mais cette hypothèse demande à être vérifiée par des travaux plus approfondis.

Des travaux antérieurs ayant montré que les plantes entières élicitées par trempage racinaire dans la fraction G5 peuvent être partiellement protégées au niveau des organes foliaires et des tiges contre une attaque par $P$. capsici (MOLOT \& MAS, 1985), on peut penser qu'il existe une relation entre cette induction systémique de résistance et le pouvoir éliciteur des extraits de plantes traitées par cette fraction. Des études se poursuivent dans ce sens.

Reçu le 13 mars 1985. Accepté le 17 octobre 1985.

RÉFÉRENCES BIBLIOGRAPHIQUES

Bailey J. A., 1974. The relationship between symptom expression and phytoalexin concentration in hypocotyls of Phaseolus vulgaris infected with Colletotrichum lindemuthianum. Physiol. Plant Pathol., 4, 477-488.

Bailey J. A., 1980. Constitutive elicitors from Phaseolus vulgaris ; a possible cause of phytoalexin accumulation. Ann. Phytopathol, 12 (4), 395-402.

Bailey J. A., 1982. Mechanisms of phytoalexin accumulation, 289312. In Bailey J. A. \& Mansfield J. W. : «Phytoalexins», Blackie, London.

Barthe J. P., 1984. La résistance du haricot à Colletotrichum lindemuthianum relevant du gène Cornell. Son expression est-elle influencée par les polygalacturonases que sécrète le parasite? Thèse Doct. d'Etat., Univ. Paul Sabatier, Toulouse, 147 p.

De Witt P. J. G. M., Spikman G., 1982. Evidence for the occurrence of race and cultivar specific elicitors of necrosis in intercellular fluids of compatible interactions of Cladosporium fulvum and tomato. Physiol. Plant Pathol., 21, 1-11.

Hahn M. G., Darvill A. G., Albersheim P., 1981. Host-pathogen interactions. XIX. The endogenous elicitor, a fragment of a plant cell wall polysaccharide that elicites phytoalexin accumulation in soybeans. Plant Physiol., 68, 1161-1169.

Hargreaves J. A., Bailey J. A., 1978. Phytoalexin production by hypocotyls of Phaseolus vulgaris in response to constitutive metabolites released by damaged bean cells. Physiol. Plant Pathol., 13, 89100 .

Hargreaves J. A., Selby C., 1978. Phytoalexin formation in cell suspension of Phaseolus vulgaris in response to an extract of bean hypocotyls. Phytochemistry, 17, 1099-1102.

Kuć J., 1982. Plant immunization. Mechanisms and practical implications, 157-178. In Wood R. K. S. : « Active Defence Mechanisms in Plants". Plenum Press New York and London, $381 \mathrm{p}$.

Keen N. T., Yoshikawa M., Wang M. C., 1983. Phytoalexin elicitor activity of carbohydrates from Phytophthora megasperma f. sp. glycinea and other sources. Plant Physiol., 71, 466-471.

Lyon G. D., Albersheim P., 1982. Host-pathogen interactions. XXI. - Extraction of a heat-labile elicitor of phytoalexin accumulation from frozen soybean stems. Plant Physiol., 70, 406-409.

Michel M. J., Molot P. M., 1980. Mise en évidence d'une induction de résistance chez Capsicum annuum par des extraits de tiges de 
piment contaminées par Phytophthora capsici. C. R. Acad. Sci. Paris, 291, ser. D, 721-723.

Molot P. M., 1984. Les phénomènes d'élicitation dans l'interaction plante-parasite fongique. Sel. fr., 34, 19-28.

Molot P. M., Mas P., 1985. Mise en évidence d'une induction de résistance chez le piment et la tomate après absorption par les racines d'une fraction élicitrice hydrosoluble. Phytopathol. Z., 112 (4), 315-321.

Molot P. M., Staron T., Mas P., 1980. La résistance du piment à Phytophthora capsici. VIII. - Induction de résistance et de capsidiol chez Capsicum annuum avec des fractions obtenues à partir du filtrat de culture et du mycélium de Phytophthora capsici. Ann. Phytopathol., 12 (4), 379-387.
Molot P. M., Mas P., Ricci P., 1982. La résistance du piment à Phytophthora capsici. XI. - Distribution spatio-temporelle du capsidiol dans les tiges infectées. Agronomie, 2 (9), 865-869.

Molot P. M., Pochard E., Mas P., 1983. La résistance du piment (Capsicum annuum) à Phytophthora capsici. XI. Réponse de 5 lignées de piment à une fraction " élicitrice ». Influence de la dose d'éliciteur et efficacité de la protection induite vis-à-vis de plusieurs souches du parasite. Agronomie, 3 (4), 327-332.

Ouchi S., 1983. Induction of resistance or susceptibility. Annu. Rev. Phytopathol., 21, 289-315. 